Sādhanā Vol. 39, Part 4, August 2014, pp. 785-808. (C) Indian Academy of Sciences

\title{
Statistically tuned Gaussian background subtraction technique for UAV videos
}

\author{
R ATHI LINGAM* and K SENTHIL KUMAR \\ Madras Institute of Technology, Chromepet, Chennai 600 044, India \\ e-mail: aathikannan@gmail.com
}

MS received 13 May 2013; revised 12 February 2014; accepted 19 May 2014;

\begin{abstract}
Background subtraction is one of the efficient techniques to segment the targets from non-informative background of a video. The traditional background subtraction technique suits for videos with static background whereas the video obtained from unmanned aerial vehicle has dynamic background. Here, we propose an algorithm with tuning factor and Gaussian update for surveillance videos that suits effectively for aerial videos. The tuning factor is optimized by extracting the statistical features of the input frames. With the optimized tuning factor and Gaussian update an adaptive Gaussian-based background subtraction technique is proposed. The algorithm involves modelling, update and subtraction phases. This running Gaussian average based background subtraction technique uses updation at both model generation phase and subtraction phase. The resultant video extracts the moving objects from the dynamic background. Sample videos of various properties such as cluttered background, small objects, moving background and multiple objects are considered for evaluation. The technique is statistically compared with frame differencing technique, temporal median method and mixture of Gaussian model and performance evaluation is done to check the effectiveness of the proposed technique after optimization for both static and dynamic videos.
\end{abstract}

Keywords. Tuning factor; background segmentation; unmanned aerial vehicle; aerial surveillance; thresholding.

\section{Introduction}

Background subtraction is a method used to segment moving objects in a video taken by a camera by comparing initial frame with the last frame. Background subtraction is a major technique in vision systems and video surveillance. In surveillance systems, the primary objective is to continuously detect the moving object in a video. In the field of aerial surveillance, it helps in understanding the movement of people to uncover suspicious events. The work is designed to process the videos captured by unmanned aerial vehicle (UAV). An unmanned aerial vehicle (commonly named as drone) is an aircraft without onboard human pilot either controlled by

*For correspondence 
computers autonomously, or under the remote control, or pilot on the ground. UAV can communicate with its controller and return electro-optic or thermal TV images payload data like position, airspeed, heading and altitude.

Since UAV captures the video dynamically, where both the object and the background keeps moving, real time systems are required to track objects effectively under such dynamic conditions. A traditional segmentation technique involves differencing two frames which works effectively on static background with fixed camera and moving object. Here, all information other than the object to be tracked remains stationary. Thus, finding difference of adjacent frames is easier. But, Aerial surveillance is a vivid environment where all the criteria are dynamic. The segmentation is done for the video captured by unmanned aerial vehicle with varying altitudes and angle of view. Here the camera is in motion to track the moving object in a moving background. Thus, traditional technique introduces ghosting effect and also the noises cannot be eliminated. This requires an adaptive measure to update the background repeatedly based on the initial frame and the last frame. This adaptive measure is implemented by a tuning factor $(\alpha)$. The optimal tuning factor ranges from 0 to 1 . The optimal value differs for static and dynamic videos. This paper involves the optimization of tuning factor that suits for both static dynamic videos. The optimization is done by evaluating the extracted statistical textures in each input frame. Based on the optimization, an adaptive Gaussian-based background subtraction technique is proposed. This running Gaussian average based background subtraction technique involves updating at both model generation phase and subtraction phase with tuning factor and Gaussian update, respectively. As only statistical properties are involved in optimizing the tuning factor and Gaussian update factor the implementation of algorithm is easier and works effectively in eliminating noise and other undesired effects. The statistical performance evaluation is done with frame differencing technique, temporal median method and mixture of Gaussian model to check the effectiveness of the proposed technique for both static and dynamic videos. The algorithms are tested with four sample videos of vivid nature like as cluttered background, small objects, moving background and multiple objects.

\section{Related work}

In this section, we review related work on various techniques involved in background subtraction of videos. Frame averaging process for colour images proposed by Anuva Chowdhury et al (2011) learns the variation of each pixel in terms of average in differences between colour of pixels. HSV-based processing is done and morphological operations were performed at the later stages. Shengyong Chen et al (2012) proposed a background segmentation method based on hierarchical model. The background update is done by mean shift technique and histogram based background subtraction. Here, the pixel descriptors were determined only for neighbouring pixels. A block based approach proposed by Weihua Xiong et al (2010) tries to overcome the drawbacks of consecutive frame subtraction by normalizing cross correlation coefficient. The non-parametric background modelling approach proposed by Martin Hofmann et al (2012) involves modelling of foreground by the history of recently accessed pixels. Shinya Miyamoori et al (2011) realized a robust approach based on bipolar radial research correlation which involves evaluation with respect to local pixels thereby suppressing the effects of illumination. Songyin Fu et al (2010) implemented background subtraction with Gaussian update and statistical model based on pixel changes. Shahrizat Shaik Mohammed et al (2010) developed a background subtraction system with mixture of Gaussians, deviation scaling factor and maxmin background model for outdoor environment. Selection of detection threshold is a difficult 


\section{VIDEO ACQUISITION -UAV}

\section{FRAME CONVERSION AND PREPROCESSING}

\section{BACKGROUND MODELLING}

\section{BACKGROUND UPDATE AND SUBTRACTION}

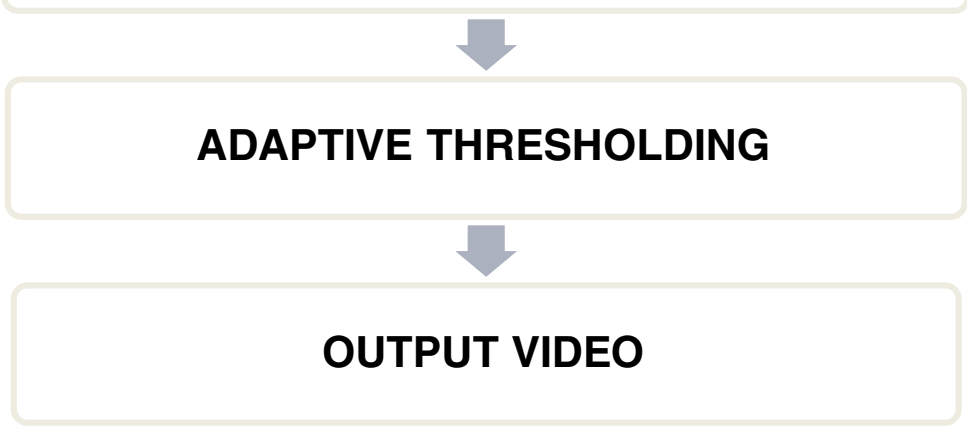

Figure 1. Workflow diagram.

task which is done easier by Syed Shazali et al (2011) by adopting the mean of video statistics. Also, a foreground model was introduced based on small spatial neighbourhood. Yongquan Xia et al (2010) proposed a Boolean based method to segment the objects with static scenes. Also, morphological processing was done for filtering and post processing.

The Gaussian mixture model of Stauffer \& Grimson (1999a, b) and kernel densities of Elgammal et al (2000) are the popular techniques for pixel level modelling. These techniques are equipped to manage slow illumination changes that can be adapted by updated model parameters.

Vijverberg et al (2009) proposed a technique to estimate the illumination change by the assumption that the foreground is very small compared to total frame size and results in a uniform histogram across the difference image by fitting Gaussian and Laplacian functions over

Table 1. Properties of input videos.

\begin{tabular}{lcccc}
\hline Property & Video 1 & Video 2 & Video 3 & Video 4 \\
\hline No of frames & 87 & 60 & 75 & 101 \\
Frame rate & $25 \mathrm{fps}$ & $25 \mathrm{fps}$ & $25 \mathrm{fps}$ & $25 \mathrm{fps}$ \\
Size & $320 \times 240$ & $320 \times 240$ & $432 \times 240$ & $432 \times 240$ \\
\hline
\end{tabular}


Table 2. Statistical computation of tuning factor.

\begin{tabular}{lllccc}
\hline Algorithm & Video & Mean & Variance & Standard deviation & Tuning factor \\
\hline FD & Video 1 & 127.95 & 6356.547 & 79.7279 & 0.738 \\
& Video 2 & 150.818 & 2408.442 & 49.076 & 0.81 \\
& Video 3 & 125.273 & 1161.160 & 34.076 & 0.89 \\
\multirow{3}{*}{ TMF } & Video 4 & 120.545 & 3394.355 & 58.261 & 0.86 \\
& Video 1 & 128.363 & 4771.194 & 69.07 & 0.55 \\
& Video 2 & 142.545 & 3026.736 & 55.016 & 0.62 \\
& Video 3 & 122.455 & 1415.879 & 37.628 & 0.68 \\
MOG & Video 4 & 119.727 & 1609.446 & 40.118 & 0.70 \\
& Video 1 & 100.591 & 5110.539 & 71.488 & 0.871 \\
& Video 2 & 150.273 & 2260.970 & 47.550 & 0.92 \\
\multirow{3}{*}{ AGBG } & Video 3 & 132.682 & 1425.465 & 37.755 & 0.846 \\
& Video 4 & 125.591 & 3789.396 & 61.558 & 0.88 \\
& Video 1 & 135.091 & 5123.039 & 71.575 & 0.602 \\
& Video 2 & 137.864 & 2772.981 & 52.659 & 0.507 \\
& Video 3 & 125.727 & 1084.398 & 32.930 & 0.498 \\
& Video 4 & 124.955 & 1786.522 & 42.267 & 0.528 \\
\hline
\end{tabular}

the histogram of the frame difference. Parameswaran et al (2010) used intensity ratios in the modelling but only as a constant with respect to spatial values. The frequently used and famous background subtraction techniques are listed and reviewed by Massimo Piccardio (2004). Sen-Ching et al (2004) discussed on probabilistic approach for robust change in shape, orientation and illumination. Also, the technique deals with detecting non-stationary objects such as swinging leaves, rain, snow and shadow cast. Olivier Barnich \& Mark Van Droogenbroeck (2011) provided a universal approach with hardware realization perspective.

Stauffer \& Grimson (1999a, b) proposed a method of defining image model consisting of description about both foreground and background values specially designed for the cases of cluttering background like snow and rain. The method proposed by Cucchiara et al (2003)

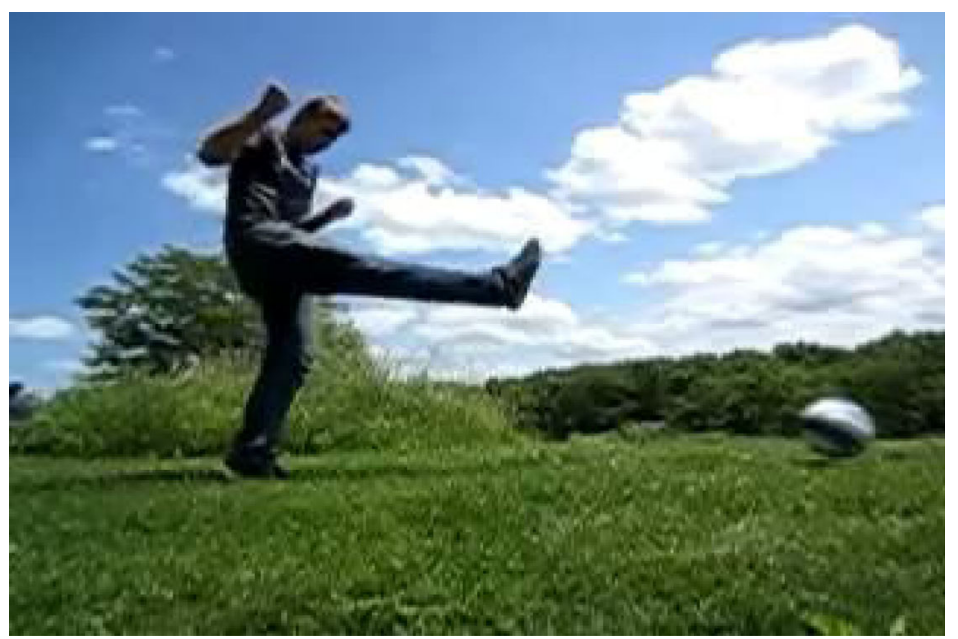

Figure 2. Video 1 of sample 1. 


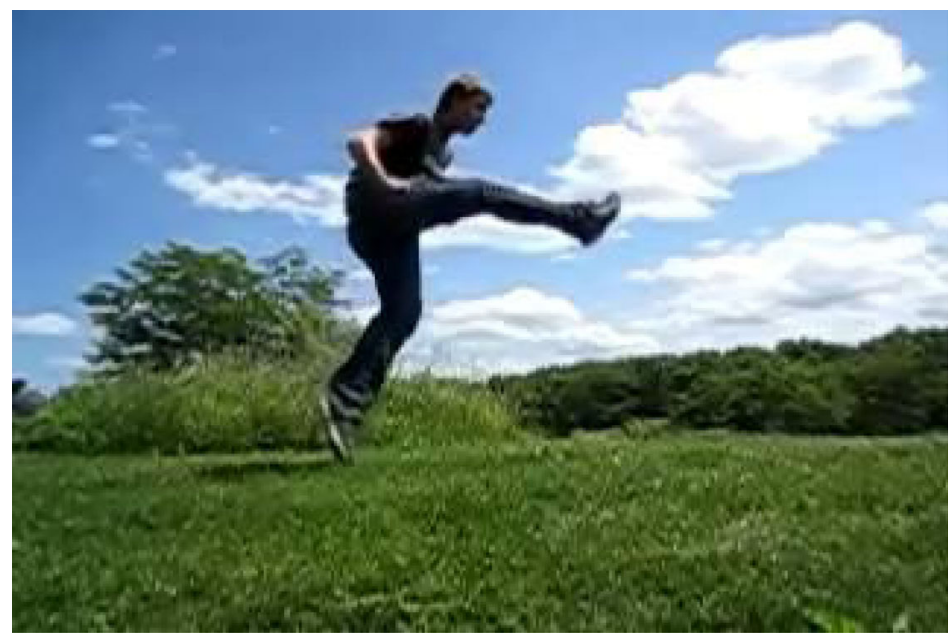

Figure 3. Video 1 of sample 2.

computes a median for special set of values. This is done to increase stability but a buffer is needed.

\subsection{Frame differencing}

Frame differencing is the basic technique which involves subtraction of adjacent frames to provide the background model.For input video of $n$ frames $F_{1}, F_{2} \ldots, F_{n}, B i$ is initial background model. The final background model $\mathrm{B}_{\mathrm{i}+1}$ is obtained by the difference between two consequent frames for $\mathrm{i}=1,2 \ldots, \mathrm{n}$

$$
\begin{gathered}
B_{i}=F_{i}, i=1, \\
B_{i+1}=F_{i+1}-F_{i},
\end{gathered}
$$

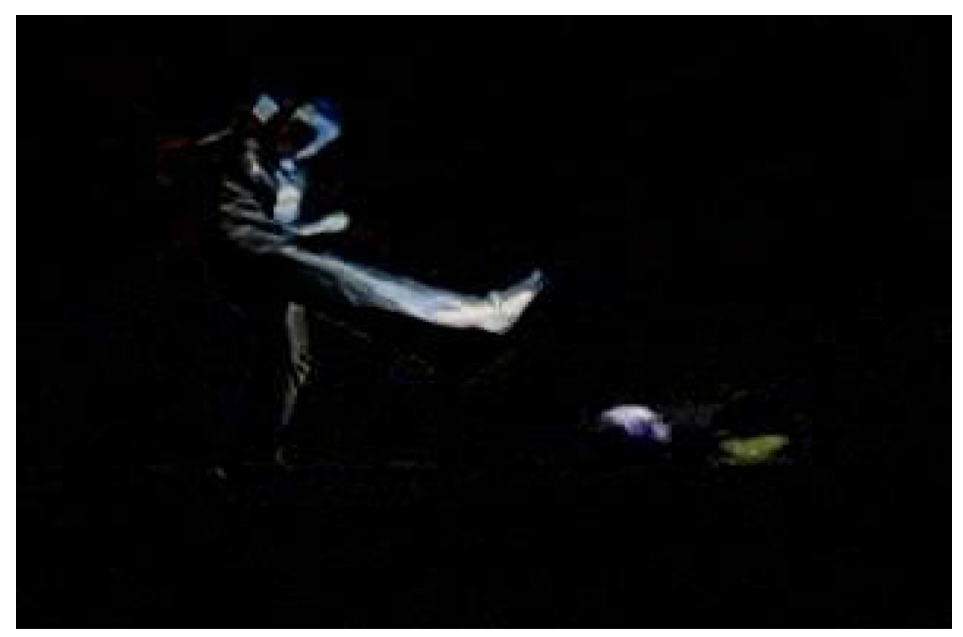

Figure 4. Video 1 of FD - BG model. 


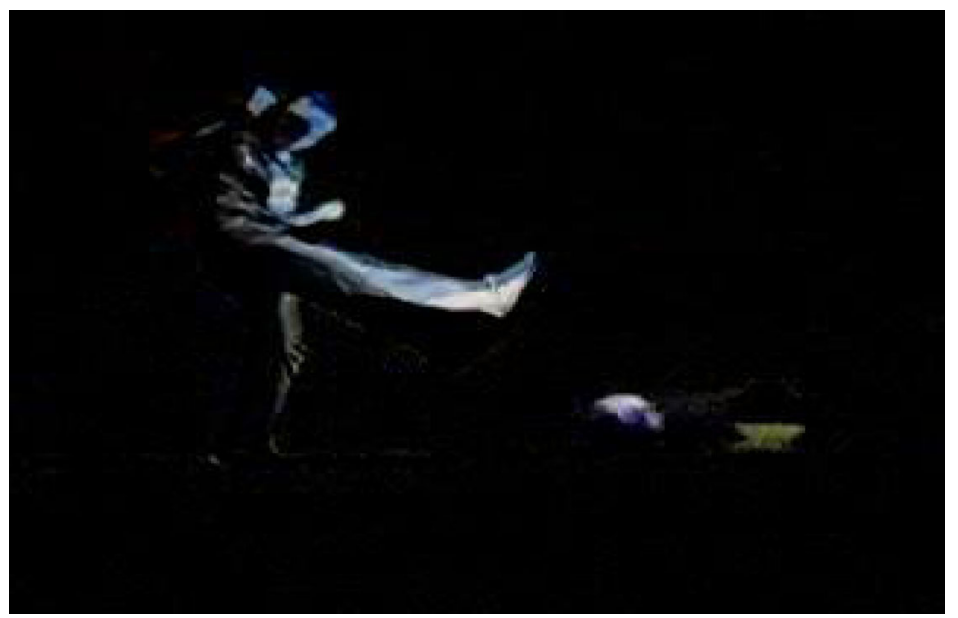

Figure 5. Video 1 of TMF - BG model.

where $F i$ is the $i^{\text {th }}$ frame and $F_{i+1}$ is the $\mathrm{i}+1^{\text {th }}$ frame. The background update $G_{i}$ is obtained by subtracting the subsequent frames $F_{i}$ with the background model $B_{i}$

$$
G_{i}=F_{i}-B_{i}
$$

The resultant binary image $\mathrm{R}_{\mathrm{i}}$ is obtained by applying the fixed threshold $\mathrm{T}$.

$$
R_{i}=\left\{\begin{array}{l}
1, G_{i}>T \\
0, G_{i}<T
\end{array}\right.
$$

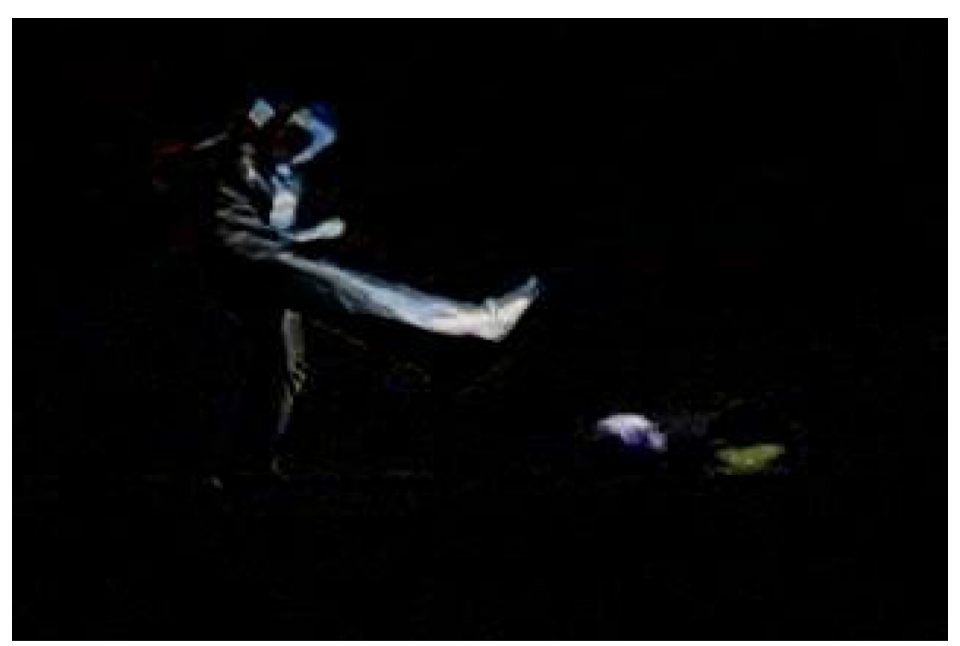

Figure 6. Video 1 of MOG - BG model. 


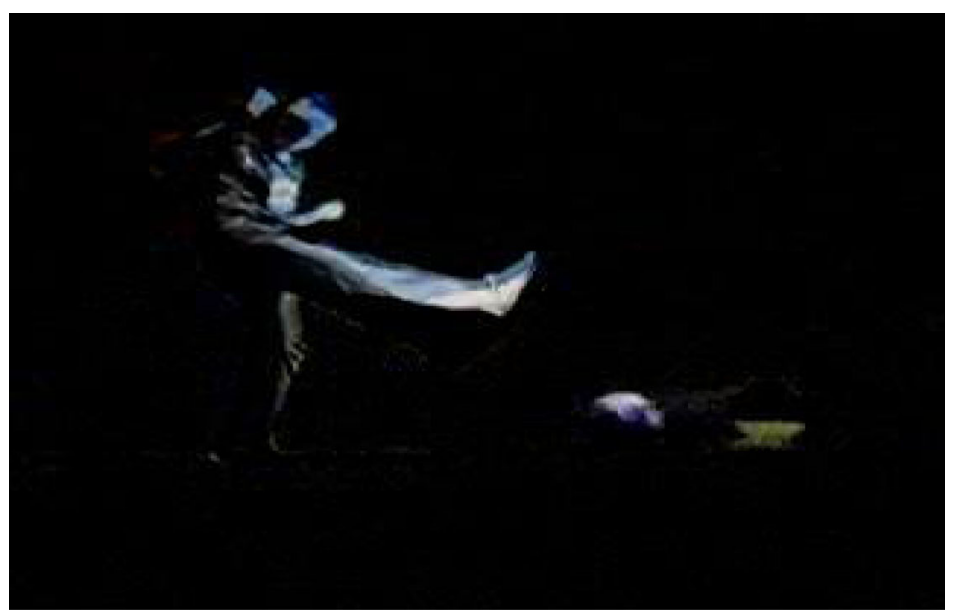

Figure 7. Video 1 of AGBG - BG model.

\subsection{Temporal median filtering}

The temporal median filtering evolves classification of target and shadows and definition of background model accordingly.

The known objects is defined as

$$
K_{0}^{t}=\left\{M V O^{t}\right\} \cup\left\{M V O_{s h}^{t}\right\} \cup\left\{G^{t}\right\} \cup\left\{G_{s h}^{t}\right\} .
$$

For the set of $S$ elements, $I^{t}(p)$ is the image pixels, $B^{t}(p)$ is the background pixel and $w_{b}$ is the weight.

$$
S=\left\{I^{t}(p), I^{t-\Delta t}(p), \ldots \ldots I^{t-n \Delta t}(p)\right\} \cup w_{b}\left\{B^{t}(p)\right\}
$$

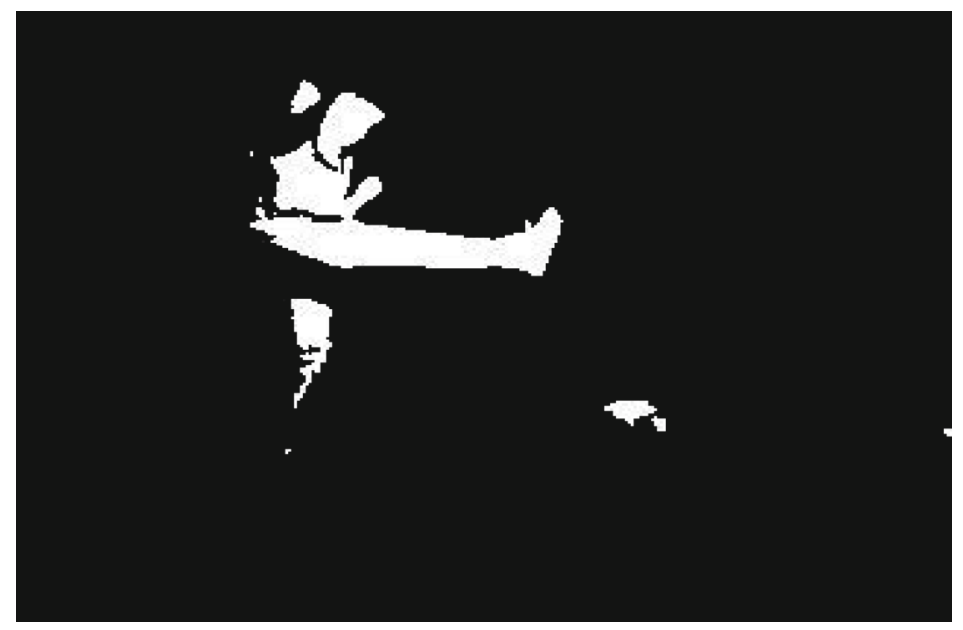

Figure 8. Video 1 of Output - FD. 


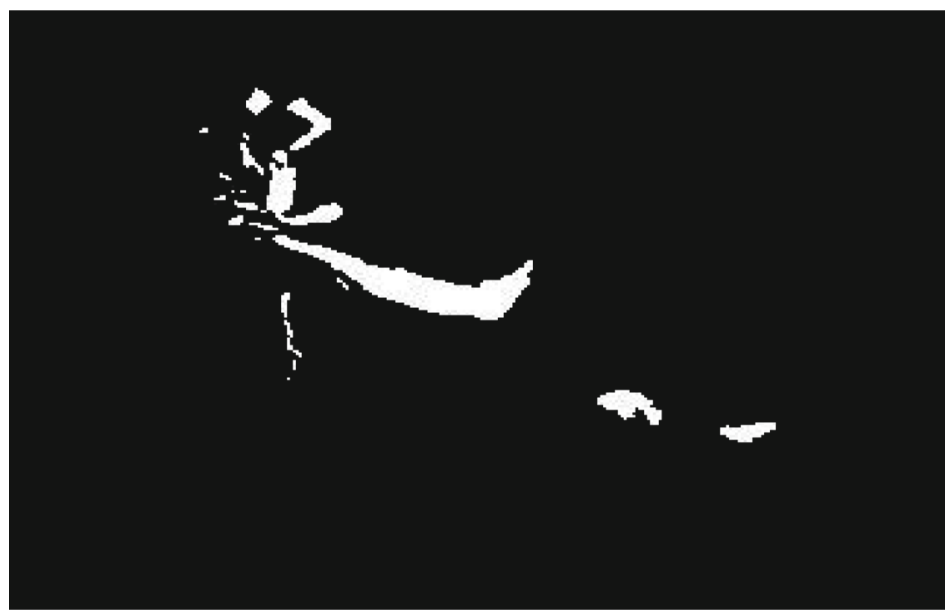

Figure 9. Video 1 of Output - TMF.

The final background model is obtained as in (7)

$$
B^{t+\Delta t}(p)=\left\{\begin{array}{c}
B_{s}^{t}(p), \text { if } p \in 0 \text { in }\left\{M V O^{t}\right\} \cup\left\{M V O_{s h}^{t}\right\} \\
B_{s}^{t+\Delta t}(p), \text { if } p \in 0 \text { in }\left\{G^{t}\right\} \cup\left\{G_{s h}^{t}\right\} .
\end{array}\right.
$$

\subsection{Mixture of Gaussians model}

Mixture of Gaussians involves multivalued background model generation for multiple background objects. It is done by defining an image model which describes both values of foreground

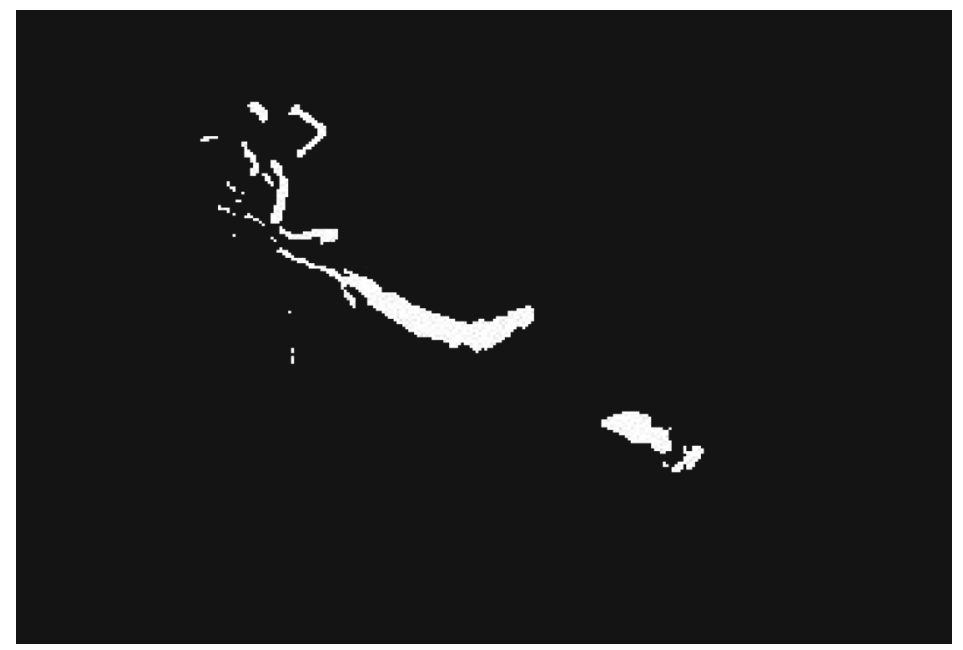

Figure 10. Video 1 of Output - MOG. 


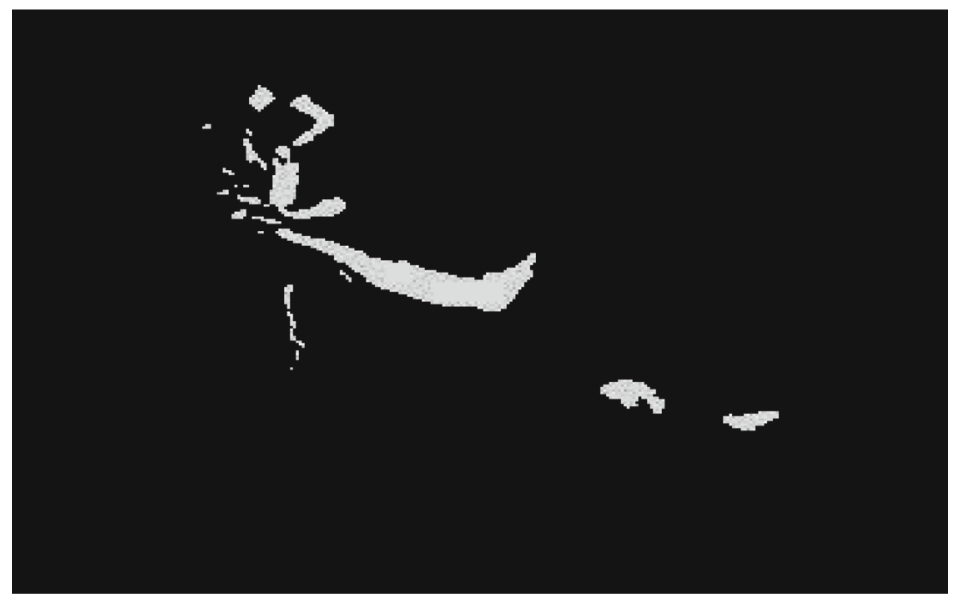

Figure 11. Video 1 of Output - AGBG.

and background based on Massimo Piccardio (2004). The probability of a pixel x at time $\mathrm{t}$ is,

$$
P\left(x_{t}\right)=\sum_{i=1}^{k} \omega_{i, t} \eta\left(x_{t}-\mu_{i, t}, \varepsilon_{i, t}\right)
$$

$\mathrm{K}$ is the Gaussian distribution ranging from 3 to 5 and $\mathrm{w}_{\mathrm{i}}$ is the peak amplitude. $\varepsilon_{\mathrm{i}, \mathrm{t}}$ is the covariance matrix. $\mu$ is the mean.

If $\mathrm{B}$ distributions are considered, the assigned threshold value $\mathrm{T}$ should satisfy,

$$
\sum_{i=1}^{B} \omega_{i}>T
$$

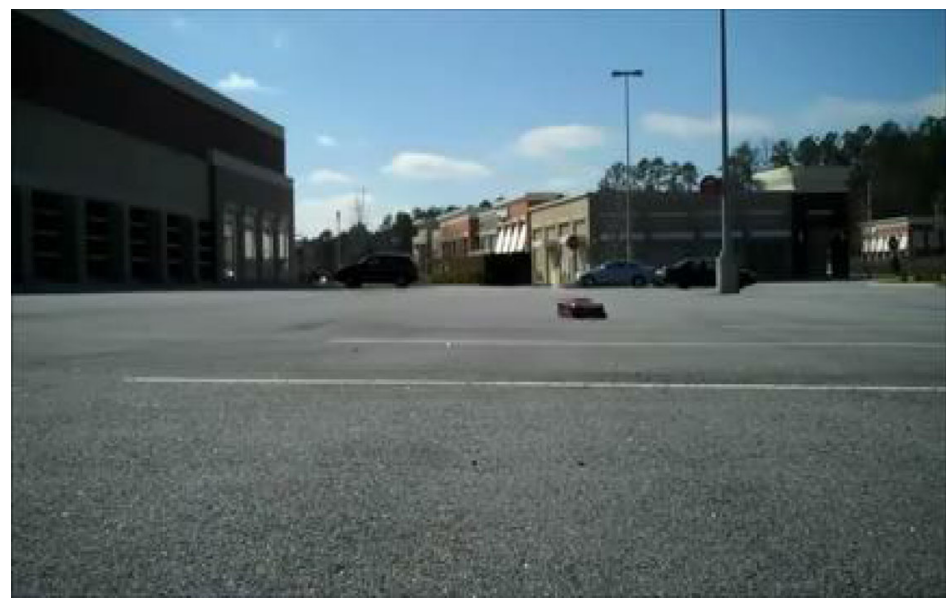

Figure 12. Video 2 of sample 1. 


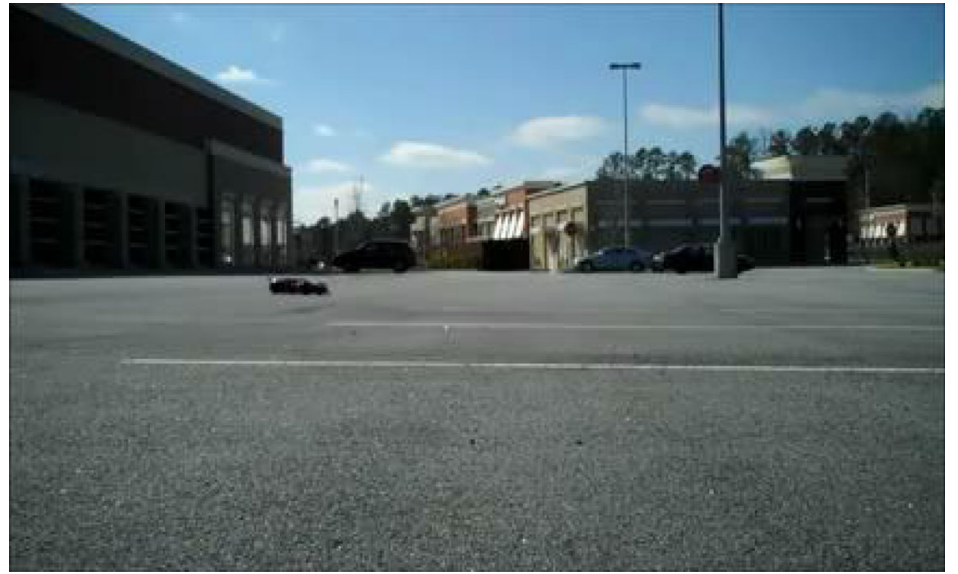

Figure 13. Video 2 of sample 2.

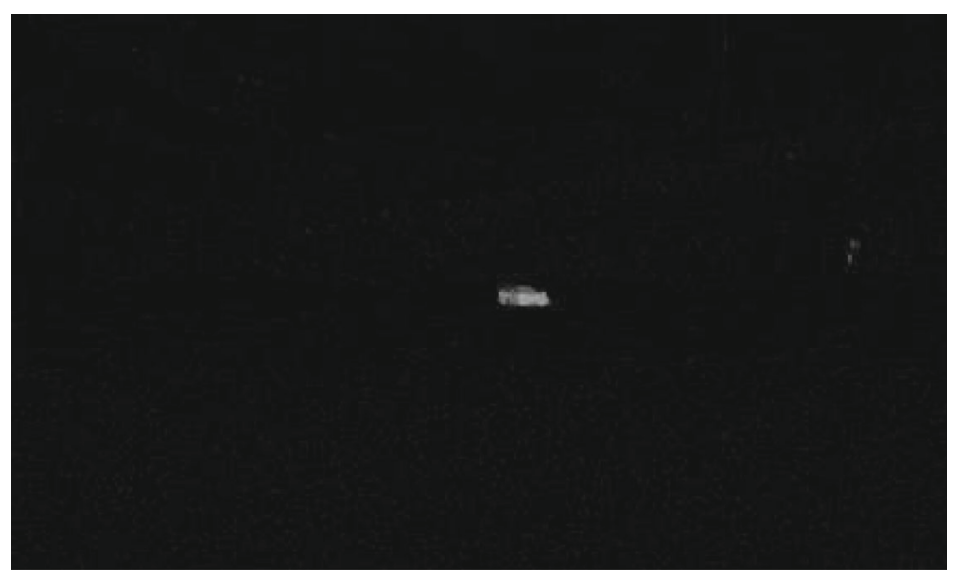

Figure 14. Video 2 of FD - BG model.

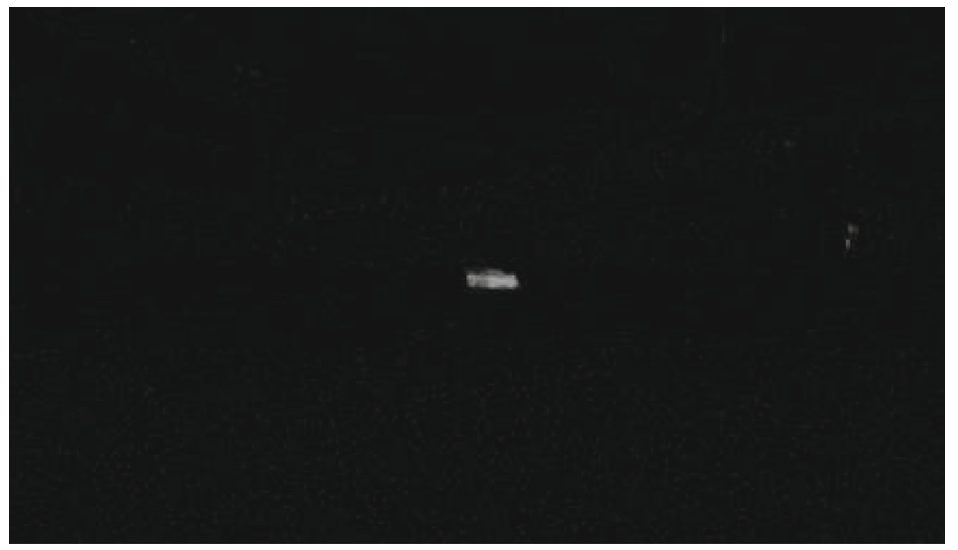

Figure 15. Video 2 of TMF - BG model. 


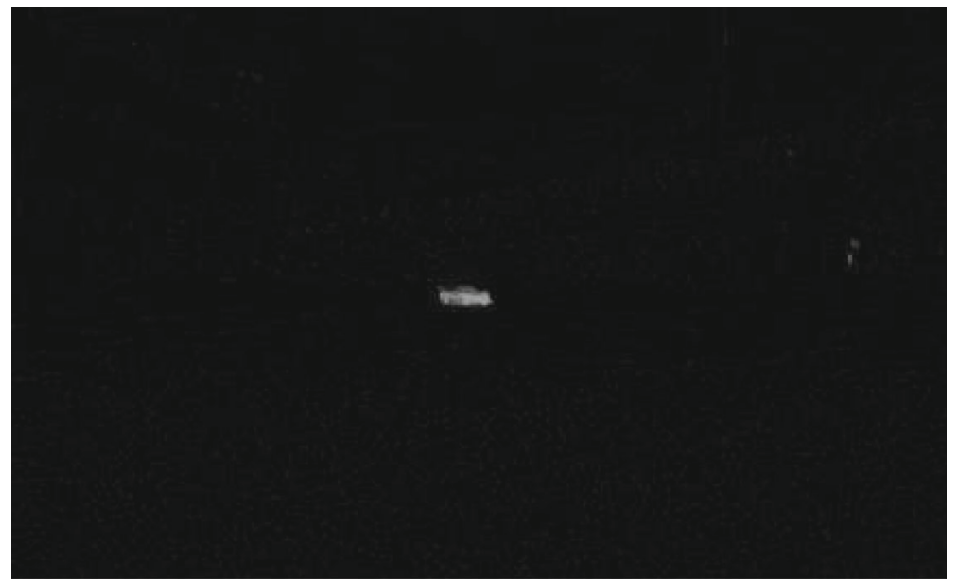

Figure 16. Video 2 of MOG - BG model.

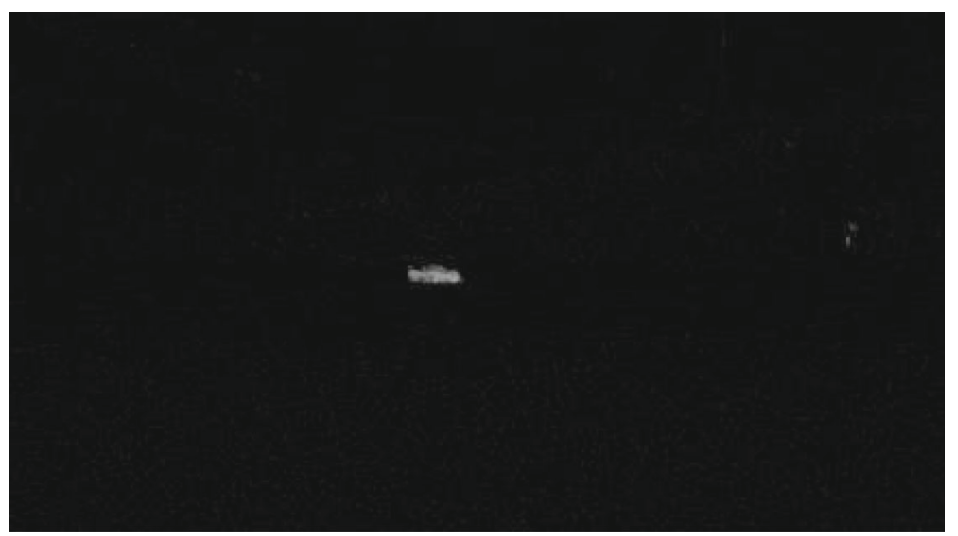

Figure 17. Video 2 of AGBG - BG model.

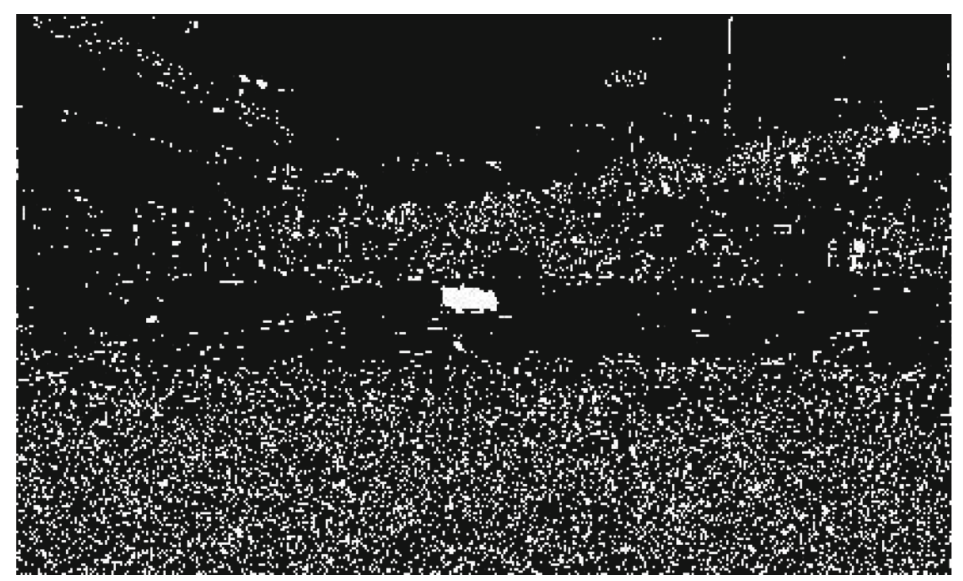

Figure 18. Video 2 of Output - FD. 


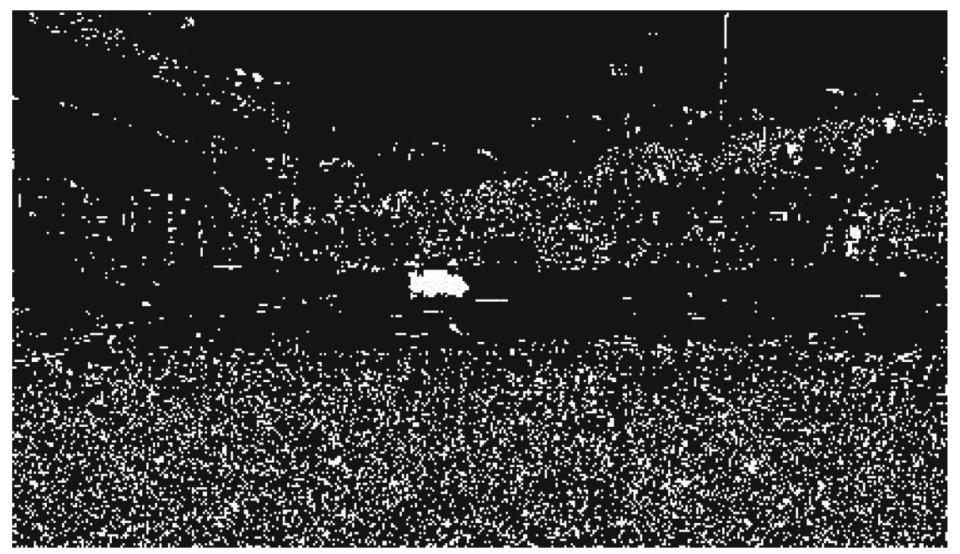

Figure 19. Video 2 of Output - TMF.

\subsection{Proposed technique - adaptive gaussian average background segmentation}

The basic idea is to subtract the input frame with the reference frame. The basic steps include background modelling, update, subtraction and thresholding. The ' $n$ ' number of frames in the video sequence is considered for analysis. The $n$ frames are named as $f_{1}, f_{2} \ldots, f_{n}$. The input frames are converted into Gray scale.

2.4a Background modelling: Background modelling is the first step in background subtraction. It constructs the reference image representing the background. Let bi be the $i^{\text {th }}$ background model. It is obtained from the input $i^{\text {th }}$ frame $f_{i}$.

At initial level $\mathrm{i}=1$ and increases linearly.

$$
b_{i}=f_{i}, i=1 \text {. }
$$

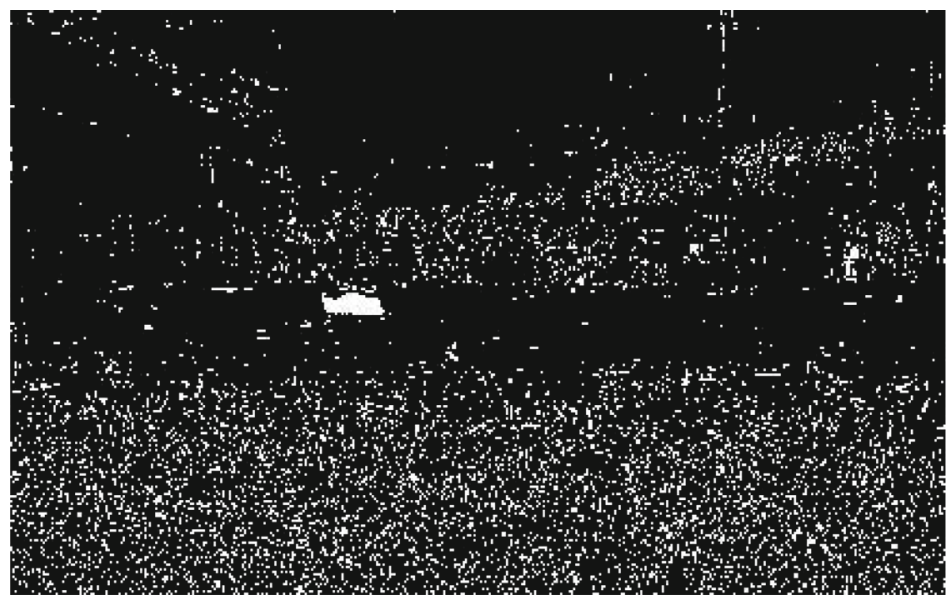

Figure 20. Video 2 of Output - MOG. 


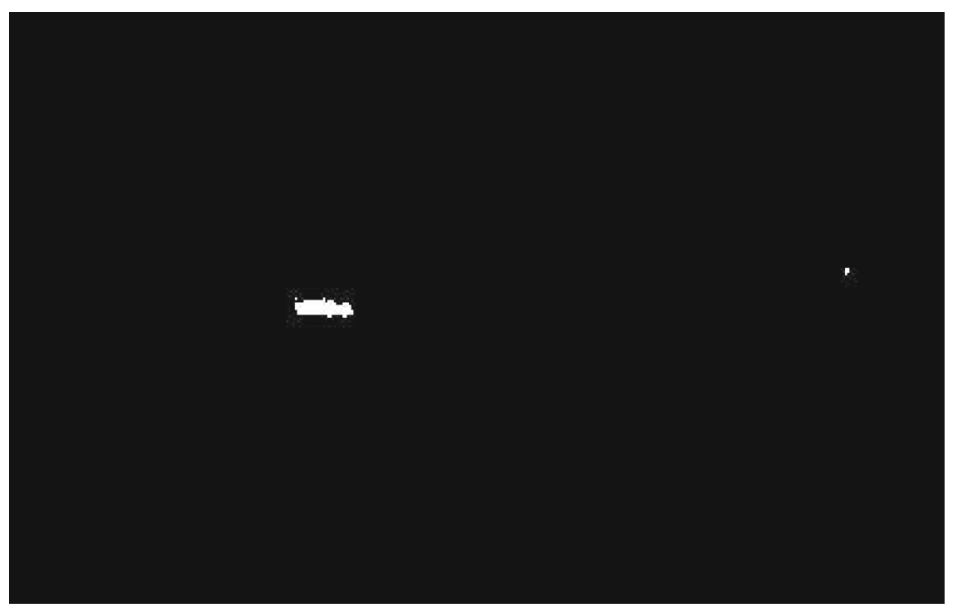

Figure 21. Video 2 of Output - AGBG.

The final background model $b_{i+1}$ is obtained by the resultant input frame fi and the assumed background model bi. Here, $i=1,2 \ldots, n$. The influence of the input frame $f_{i}$ and the priory assumed background model bi are proportioned with the tuning factor $\alpha$. The value of $\alpha$ decides the extent that both the frames can influence in the next background model. In general $\alpha$ varies from 0 to 1 .

$$
b_{i+1}=\propto f_{i}+(1-\propto) b_{i} .
$$

If $\alpha=0$, then bi $+1=$ bi, thus there will be no updation in background model. This results in ghosting effect. If $\alpha=1$, then ${ }_{\mathrm{bi}+1}=\mathrm{f}_{\mathrm{i}}$. Hence the background model and the input frame are same and the result reduces to Boolean 0 . Thus it is necessary to update the tuning factor to get the desired result.

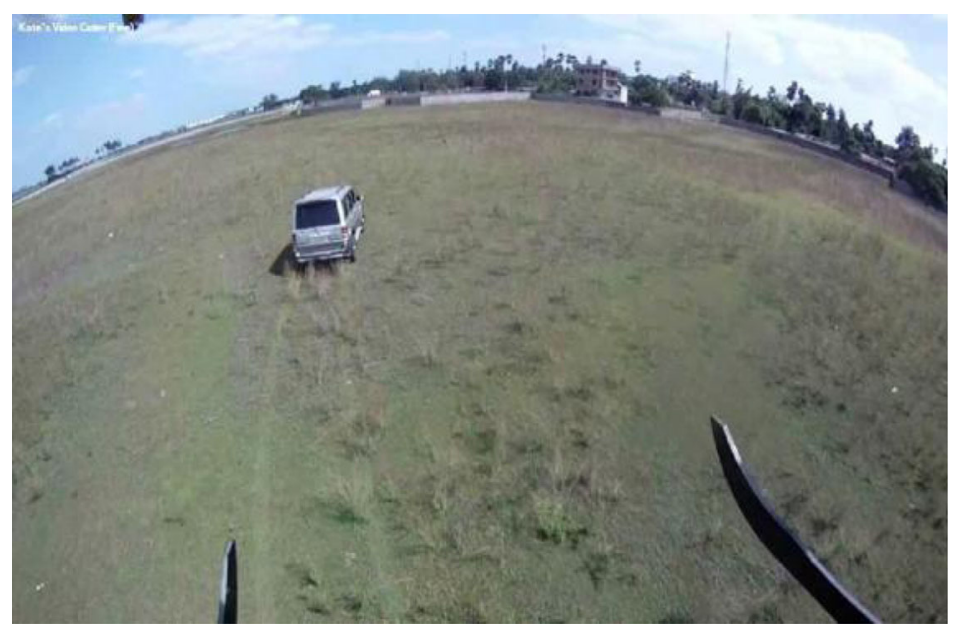

Figure 22. Video 3 of sample 1. 


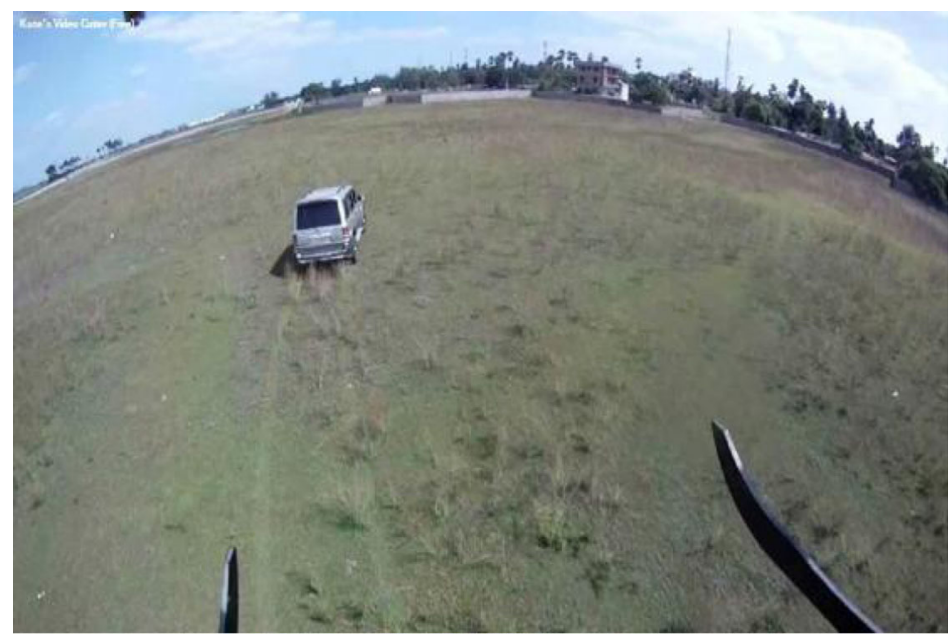

Figure 23. Video 3 of sample 2.

2.4b Background update: Let the background update be $\mathrm{g}_{\mathrm{i}}$. It is obtained by subtracting the subsequent input frames $f_{i}$ with the tuned background model $b_{i}$.

$$
g_{i}=f_{i}-b .
$$

2.4c Thresholding: Thresholding is a process used to classify the input pixels into background or object based on the value assumed as threshold 'Th'. Generally, the pixels with values above 'Th' are considered as foreground and the pixels with values below Th are considered as background. The fixed threshold will not produce efficient segmentation for various lighting

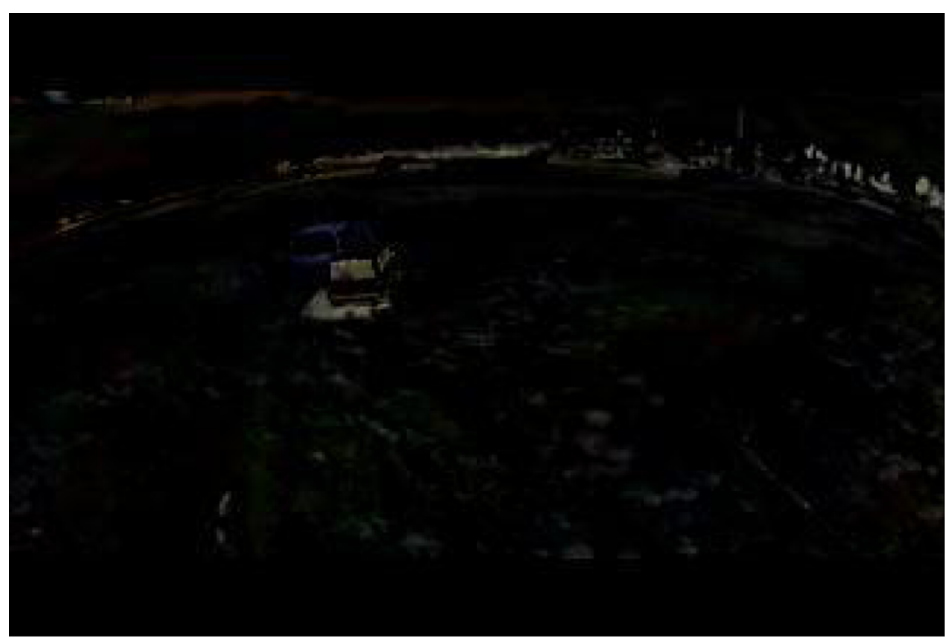

Figure 24. Video 3 of FD - BG model. 


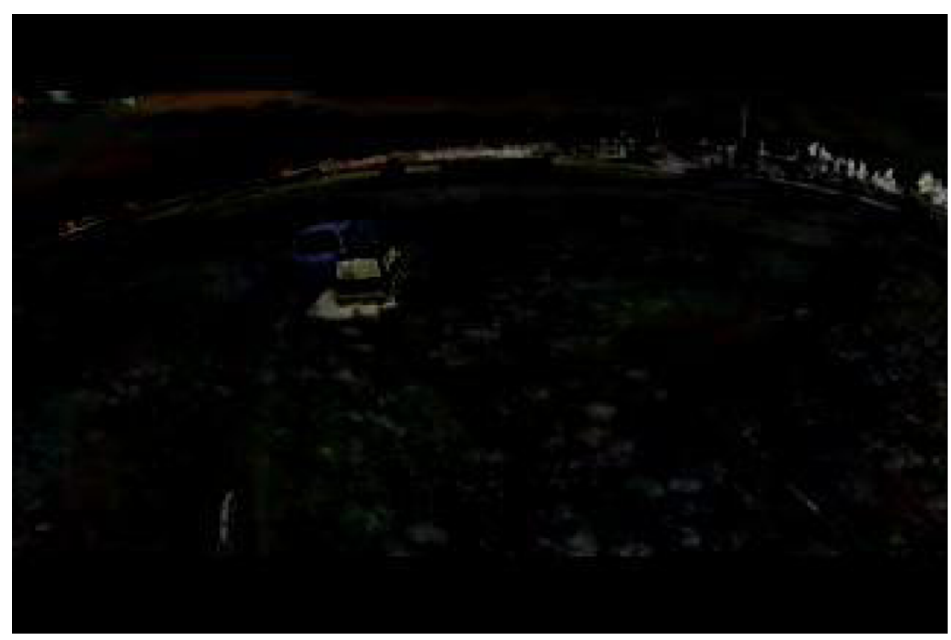

Figure 25. Video 3 of TMF - BG model.

conditions. Thus, we consider an adaptive approach to update the threshold value based on the statistical properties of the input frame and background model. The resultant binary image $r_{i}$ is obtained based on the value of threshold Th.

$$
r_{i}= \begin{cases}1, & g_{i}>T h \\ 0, & g_{i}<T h\end{cases}
$$

The adaptive threshold is decided as the average standard deviation of each video frame. The value of $\sigma$ is updated for each frame.

$$
\begin{gathered}
\text { Th=mean }(\sigma), \\
\sigma_{i+1}^{2}=\alpha\left(f_{i}-b_{i}\right)^{2}+(1-\alpha) \sigma_{i}^{2} .
\end{gathered}
$$

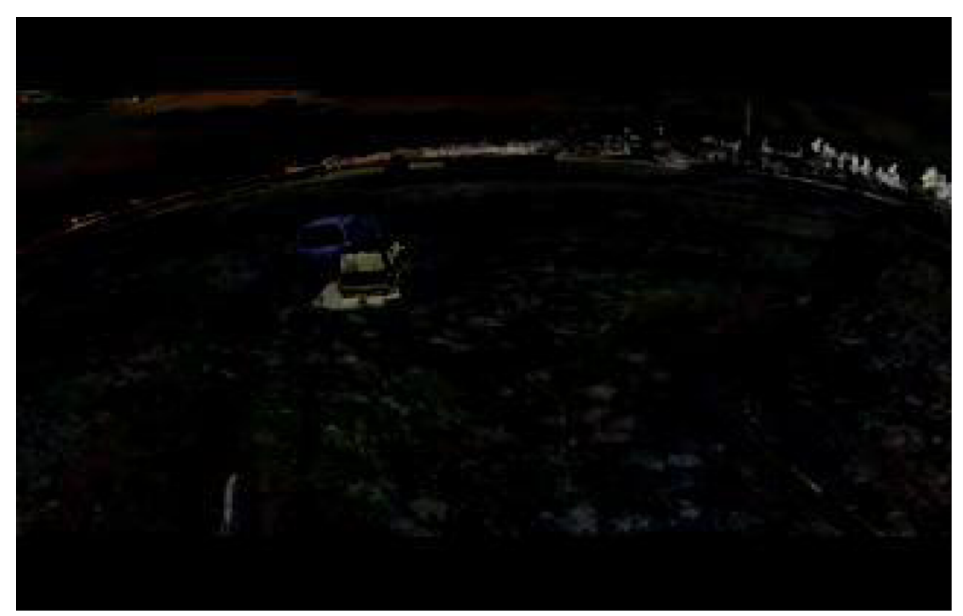

Figure 26. Video 3 of MOG - BG model. 


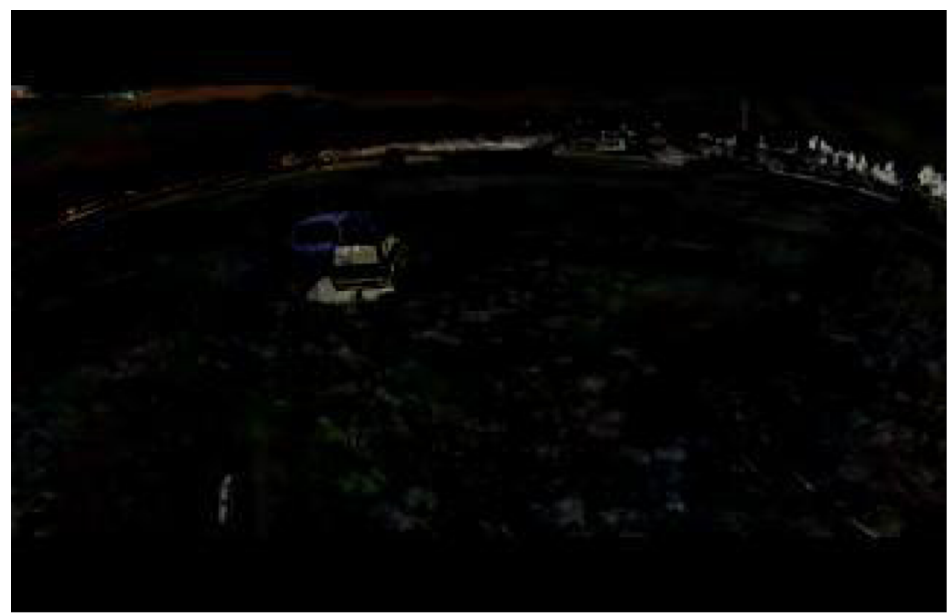

Figure 27. Video 3 of AGBG - BG model.

\section{Optimization of tuning factor}

Optimization of the tuning factor $\alpha$ is very important in background modelling and update. Since in aerial videos both the object and background are dynamic, the traditional process of subtracting two subsequent frames will not be effective. Thus, to classify the background and object it is necessary to obtain the statistical features of the image. The statistical properties of the object differ widely from the background. Let $\mathrm{X}_{\mathrm{ij}} \mathrm{n}$ be the gray scale vector for the pixel location $(\mathrm{i}, \mathrm{j})$ for $\mathrm{n}$ input frames. With $\mathrm{E}[$.$] as expectation function, \mathrm{E}_{\mathrm{ij}}\left[\mathrm{X}_{\mathrm{ij}} \mathrm{n}\right]$ is the mean of a vector of grayscale pixel intensity at pixel location (i,j) for $n$ frames. The tuning factor $\alpha$ is obtained as

$$
\propto_{i j}(n)=\left\{X_{i j} n-E_{i j}\right\} U_{x}
$$

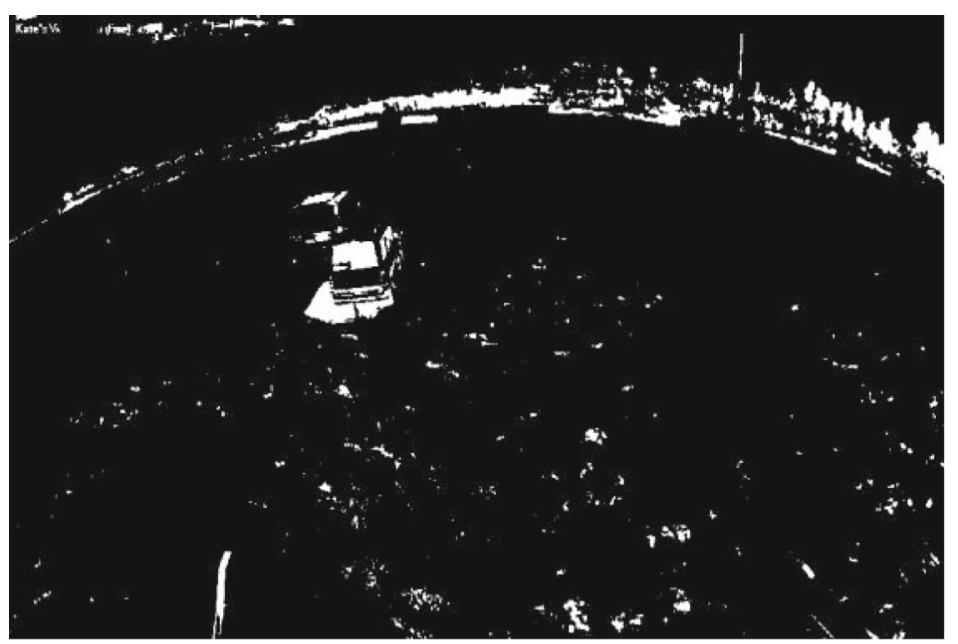

Figure 28. Video 3 of Output - FD. 


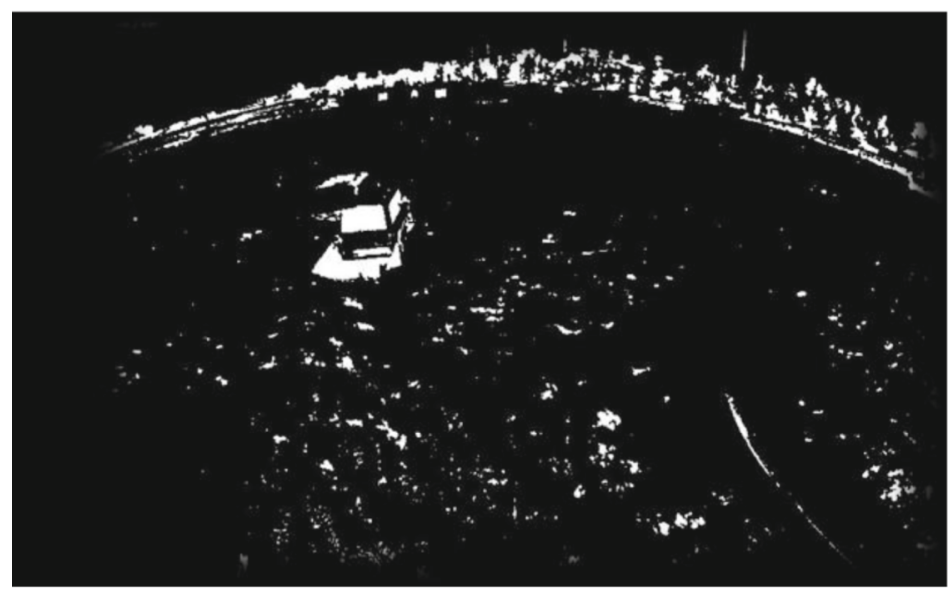

Figure 29. Video 3 of Output - TMF.

$\mathrm{X}_{\mathrm{ij}} \mathrm{n}$ is the pixel intensity of $\mathrm{nth}$ frame at location $(\mathrm{i}, \mathrm{j}) . \mathrm{E}_{\mathrm{ij}}$ is the mean of intensities of $\mathrm{n}$ frames at location $(i, j)$. By evaluating the variance and standard deviation for each frame, and by normalizing

$$
\propto=\left(X_{i j} n-E_{i j}\right) / \sigma_{i j} .
$$

The value of $\alpha$ is optimized and varies from 0 to 1 for both static and dynamic background models.

\section{Implementation}

Four videos are considered for analysis. Two of them are with static background and the other two are with dynamic background taken from UAV. The videos with dynamic background are

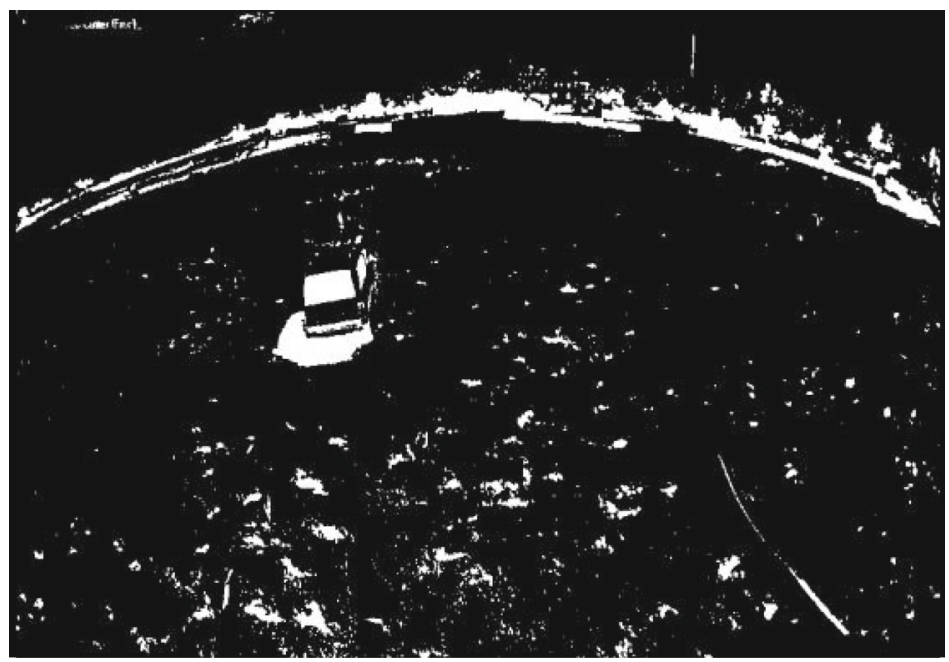

Figure 30. Video 3 of Output - MOG. 


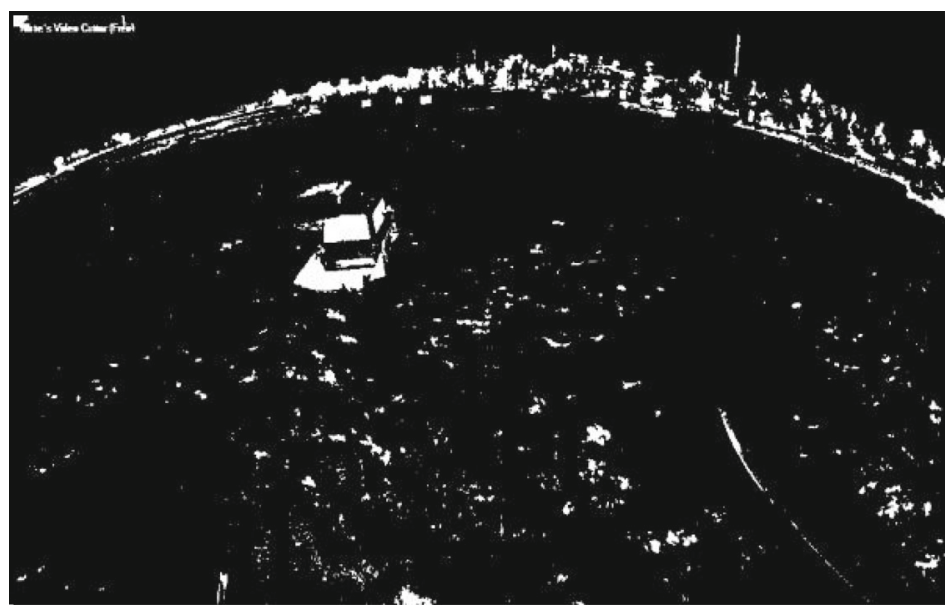

Figure 31. Video 3 of Output - AGBG.

transmitted with the transmission frequency of $1.2 \mathrm{GHz}$ from UAV with altitude ranging from 5 to $50 \mathrm{~m}$. The properties of the videos are listed in the table 1 .

\section{Results and discussion}

The output is obtained by simulation using MATLAB 2010 in a standalone PC with 4 GB Intel Core Duo processor. In each video, 25 frames are considered for analysis. In each frame 100 pixels at random locations are considered and their intensities are taken for processing. Based on the considered pixel values, the statistical calculations are made to determine mean, standard deviation and variance. Based on these three factors, the tuning factor is determined. Table 2 shows the statistical values obtained for videos with static background and videos with dynamic background for the four algorithms considered.

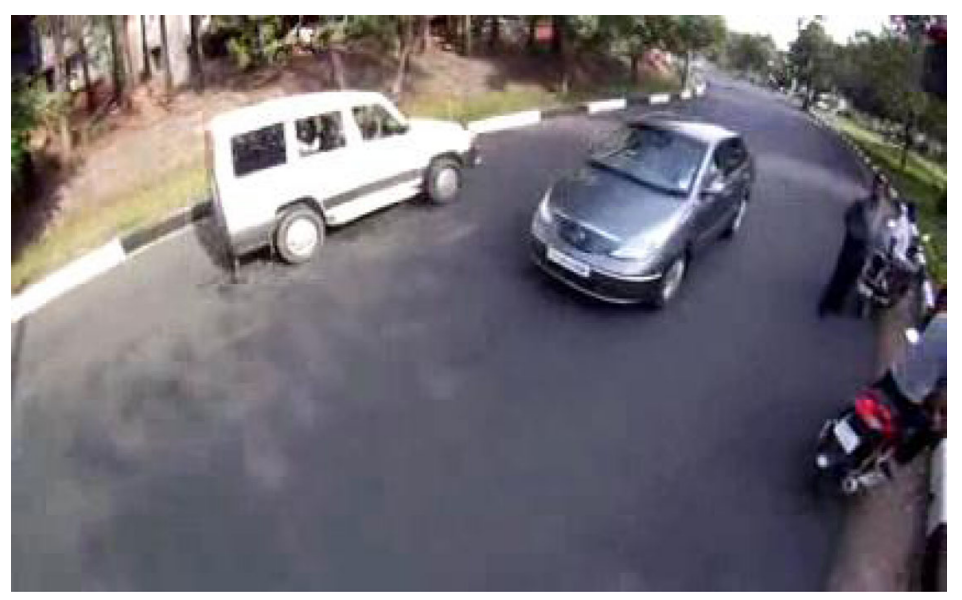

Figure 32. Video 4 of sample 1. 


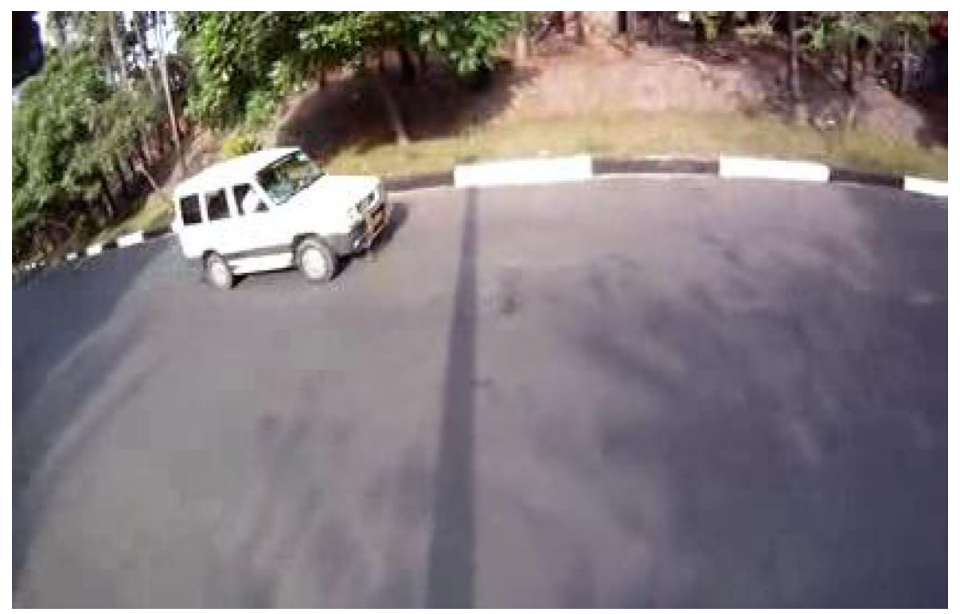

Figure 33. Video 4 of sample 2.

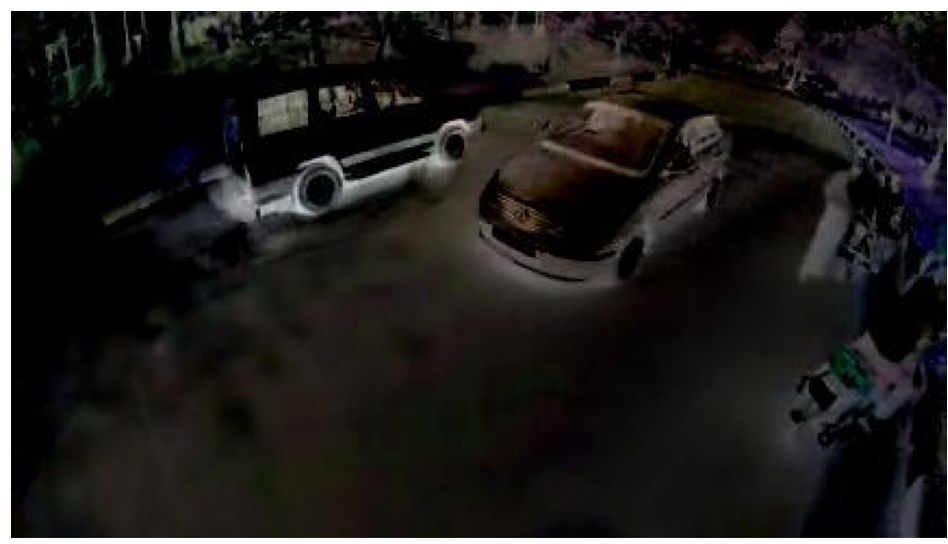

Figure 34. Video 4 of FD - BG model.

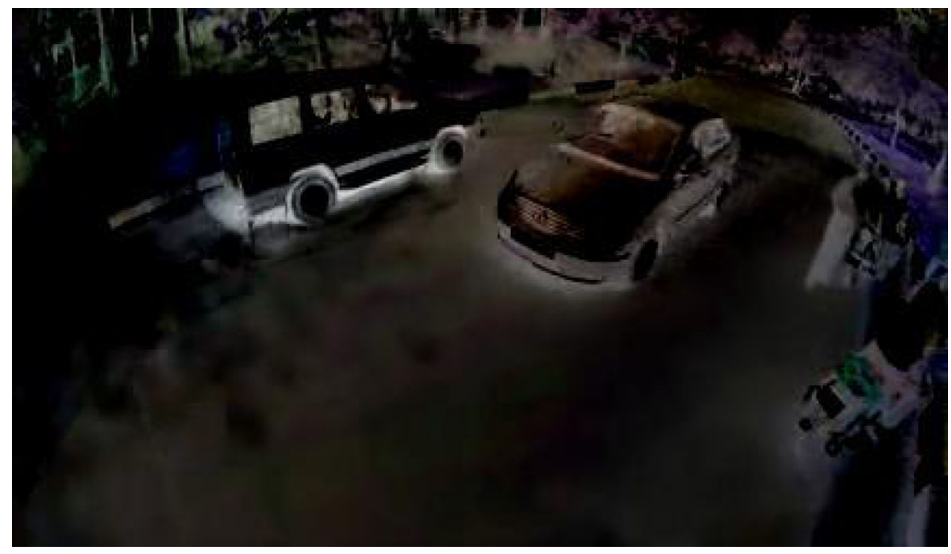

Figure 35. Video 4 of TMF - BG model. 


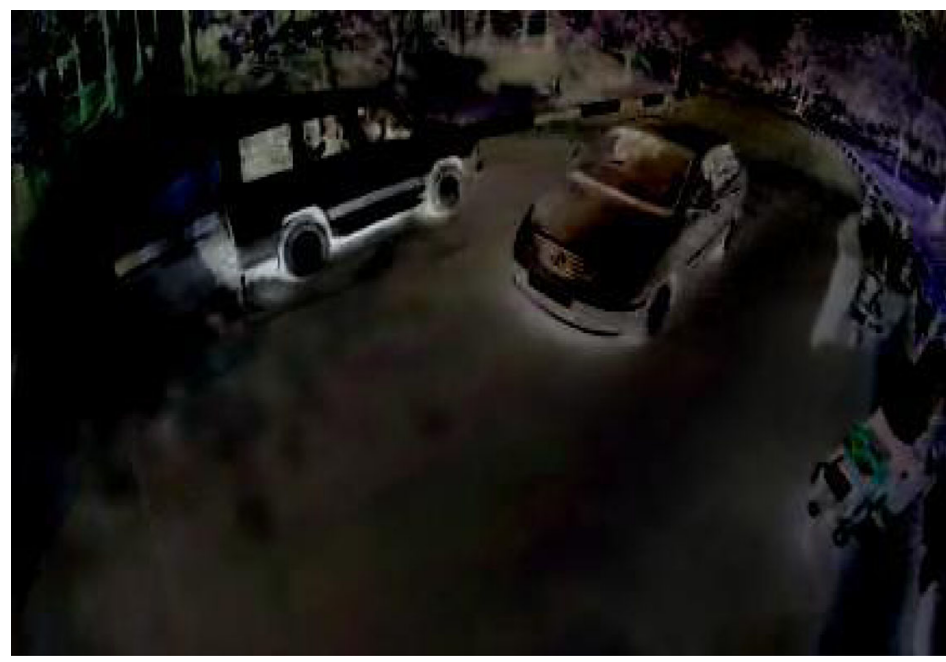

Figure 36. Video 4 of $\mathrm{MOG}-\mathrm{BG}$ model.

In table 2, for the algorithm adaptive Gaussian background subtraction the average tuning factor for video 1 is 0.602 , for video 2 is 0.507 , for video 3 is 0.498 and for video 6 is 0.528 . These values are the average of a sample of 25 frames in each video. The overall tuning factor is approximately around 0.53 . Thus, while defining the value for $\alpha$, it may be assigned varying range from 0.5 to 0.6 for efficient segmentation.

Based on the tuning factor and Gaussian update derived, the running Gaussian averaging background segmentation is implemented for the static and dynamic background videos. Also, the results of existing techniques like frame differencing, temporal median filter and mixture of

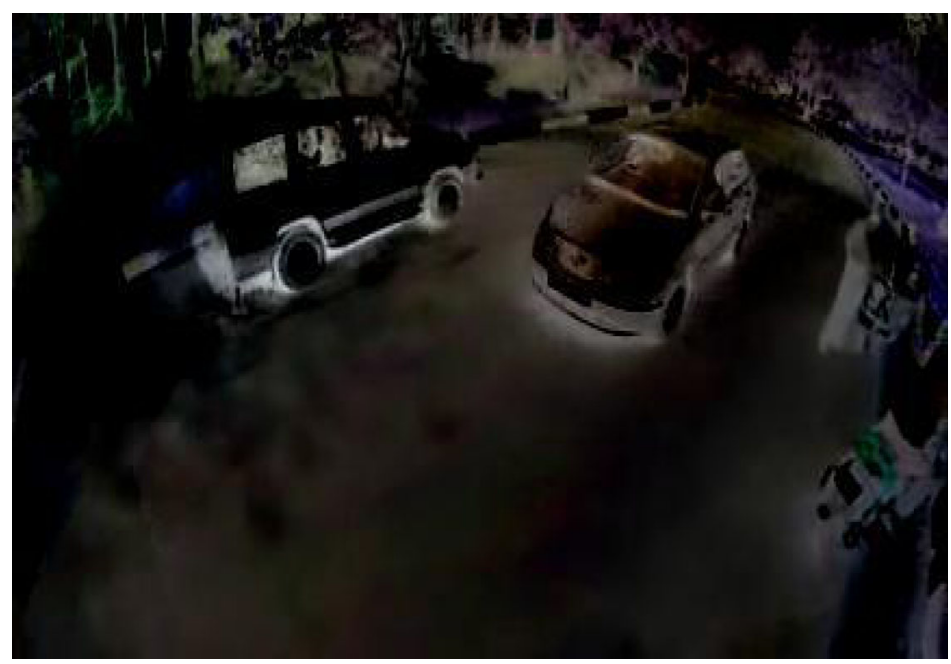

Figure 37. Video 4 of AGBG - BG model. 


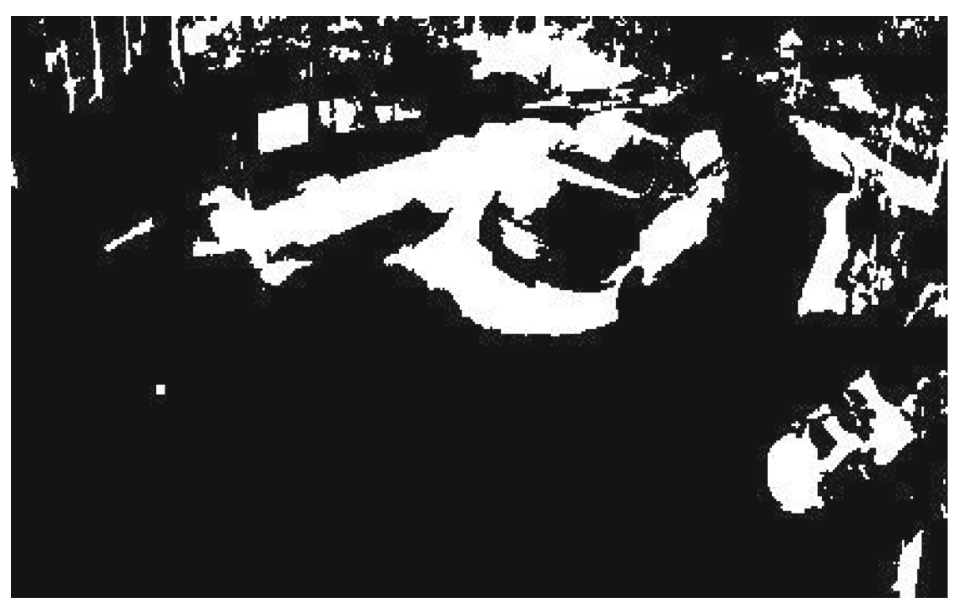

Figure 38. Video 4 of Output - FD.

Gaussian models are obtained. The optimization of tuning factor is not suitable for other techniques since the value $\alpha$ is not used. The tuning factor is optimized to improve the efficiency of the proposed techniques compared to others.

Four videos are considered for analysis (figures 1). Video 1 (figures 2 and 3) consists of static background and the target is larger in size. The corresponding background models are shown in (figures 4-7) and the final subtracted images are shown in (figures 8-11). Video 2 (figures 12 and 13) consists of static background and the target is smaller in size. The background is cluttered and consists of overlapping information. The corresponding background models are shown in (figures 14-17) and the final subtracted images are shown in (figures 18-21). Video 3 (figures 22 and 23) consists of dynamic background and the target is larger in size. There is a single target in the video. Both the background and the target are moving. The corresponding background models are shown in (figures 24-27) and the final subtracted images are shown in (figures 28-31). Video 4 (figures 32 and 33) consists of dynamic background and the target is larger in size. There

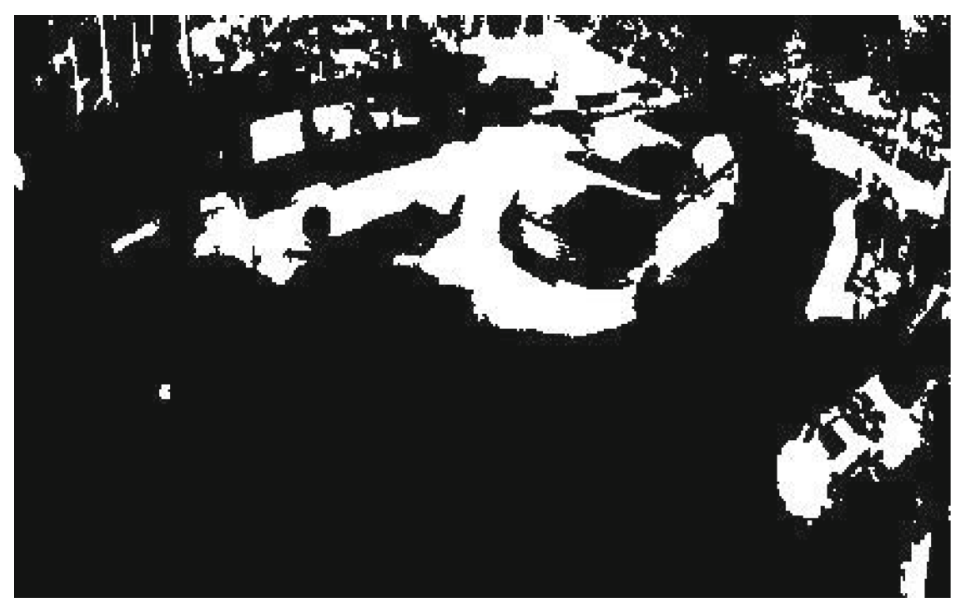

Figure 39. Video 4 of Output - TMF. 


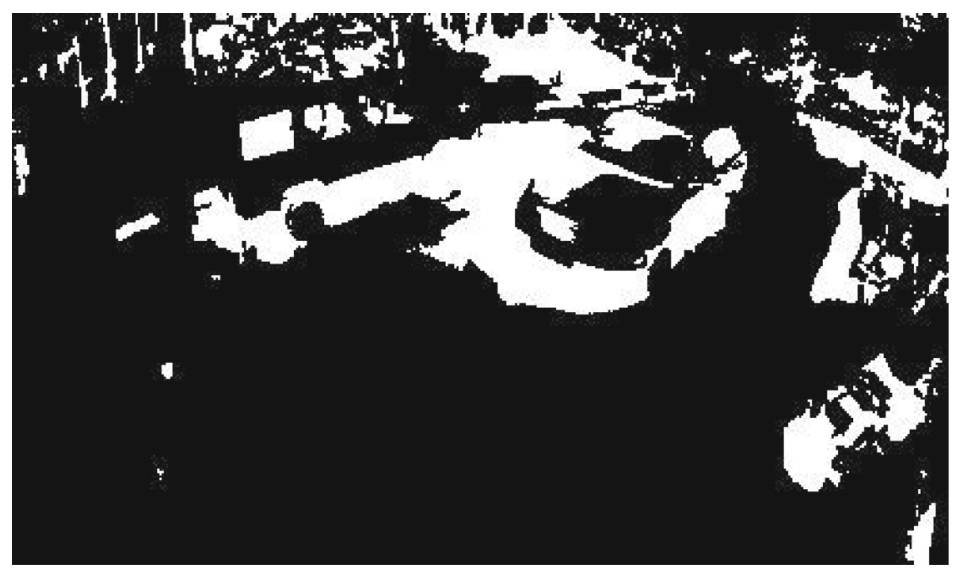

Figure 40. Video 4 of Output - MOG.

are multiple moving objects in the video. The background is cluttered. Both the background and the target are moving. The corresponding background models are shown in (figures 34-37) and the final subtracted images are shown in (figures 38-41).

To evaluate the effectiveness of the proposed algorithm tuned with $\alpha$ with respect to the other algorithms, some performance evaluation parameters like Peak Signal to Noise Ratio, Root Mean Square Error and Universal Image Quality Index of the input and output were calculated.

The Root Mean Square Error is calculated as,

$$
R M S E=\sqrt{\frac{1}{M N} \sum_{j=1}^{M} \sum_{k=1}^{N}\left(x j k-x^{\prime} j k\right)^{2}},
$$

where, $\mathrm{M}$ and $\mathrm{N}$ are the total pixels to be considered.

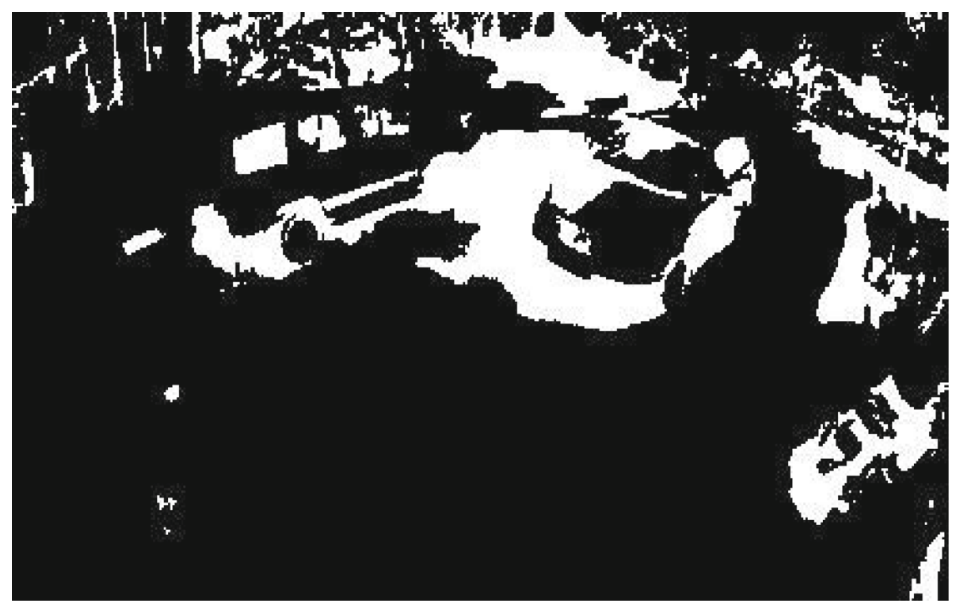

Figure 41. Video 4 of Output - AGBG. 
Table 3. Statistical features of output frames.

\begin{tabular}{lccccc}
\hline Algorithm & Video & MSE & RMSE & PSNR & UIQI \\
\hline FD & Video 1 & 139.719 & 11.820 & 26.678 & 0.1668 \\
& Video 2 & 84.490 & 9.19 & 28.863 & 0.0564 \\
& Video 3 & 126.93 & 11.266 & 27.095 & 0.1193 \\
\multirow{3}{*}{ TMF } & Video 4 & 127.125 & 11.231 & 27.088 & 0.144 \\
& Video 1 & 138.624 & 11.774 & 26.712 & 0.171 \\
& Video 2 & 83.954 & 9.163 & 28.89 & 0.614 \\
& Video 3 & 126.524 & 11.248 & 27.109 & 0.119 \\
\multirow{3}{*}{ MOG } & Video 4 & 126.982 & 11.268 & 27.093 & 0.152 \\
& Video 1 & 138.126 & 11.753 & 26.72 & 0.189 \\
& Video 2 & 83.126 & 9.117 & 28.933 & 0.686 \\
& Video 3 & 126.114 & 11.23 & 27.123 & 0.123 \\
AGBG & Video 4 & 126.148 & 11.232 & 27.122 & 0.175 \\
& Video 1 & 137.229 & 11.714 & 26.756 & 0.193 \\
& Video 2 & 82.483 & 9.08 & 28.967 & 0.072 \\
& Video 3 & 125.652 & 11.209 & 27.139 & 0.123 \\
& Video 4 & 125.534 & 11.204 & 27.143 & 0.185 \\
\hline
\end{tabular}

The Peak signal to Noise ratio is the commonly used performance measure and it is evaluated as

$$
P S N R=10 \log 255^{2} / M S E .
$$

The universal image Quality Index $(\mathrm{Q})$ is calculated for the series of input images $X=\mathrm{x}_{\mathrm{i}}$, and output frame $\mathrm{y}_{\mathrm{i}}$. The range of ' $\mathrm{i}$ ' varies from 1 to $\mathrm{N}$, where $\mathrm{N}$ is the total number of frame pairs considered for analysis.

$$
Q=\frac{4 \sigma x y \cdot X Y}{\left(\sigma x^{2}+\sigma y^{2}\right)\left(X^{2}+Y^{2}\right)},
$$

$\sigma_{x y}$ is the cross correlation, $\sigma_{\mathrm{x}}$ and $\sigma_{\mathrm{y}}$ are the correlation of $\mathrm{x}$ and $\mathrm{y}$, respectively. $\mathrm{X}$ and $\mathrm{Y}$ are the mean value of $x$ and $y$, respectively.

In each video, 25 frames are considered for evaluation and the average performance values are listed in table 3.

The table 3 shows that the algorithm tuned with $\alpha$ provides effective segmentation. More over the computation time is less. The quality of segmentation is good comparatively as the signal to noise ratio is high and the mean square error is less. Rather than other techniques, the algorithm suits better for aerial surveillance applications due to high speed of computation since the complexity is less.

\section{Conclusion}

We have portrayed an efficient algorithm with tuning factor and Gaussian update for aerial surveillance. The optimization of tuning factor is done by extracting the statistical features of the input frames. The optimized value of tuning factor is found to be 0.5 to obtain efficient segmentation in adaptive Gaussian background subtraction. The statistical analysis depicts that the algorithm when added with tuning factor is efficient for both static and dynamic videos. It works efficiently for videos which are of dynamic nature completely. Since aerial videos are of that category the proposed technique works dynamically with less computational complexity and provides good quality of segmentation thus can be utilized for real time tracking. 


\section{References}

Anuva Chowdhury, Sang Jin Cho and Vi Pil Chong 2011 A background subtraction method using color information in the framing averaging process, The $6^{\text {th }}$ International forum on Strategic Technology

Cucchiara R, Grana C, Piccardi M and Prati A 2003 Detecting moving objects, ghosts and shadows in video streams. IEEE Trans. Pattern Anal. Mach. Intell. 25(10): 1337-1342

Elgammal A M, Harwood D and Davis L S 2000 Non-parametric model for background subtraction. In: Proceedings of the 6th European Conference on Computer Vision-Part II, ECCV '00, 751-767, London, UK: Springer

Martin Hofmann, Philipp Tiefenbacher and Gerhard Rigoll 2012 Background segmentation with feedback: The pixel based adaptive segmenter. IEEE Computer Vision and Pattern Recognition Workshop, 16-12 June, 38-43

Massimo Piccardio 2004 Background Subtraction Techniques: A Review. IEEE Int. Conf. Syst. Man Cybern. 3099-3104

Olivier Barnich and Mark Van Droogenbroeck 2011 VIBE: A universal background subtraction algorithm for video sequences. IEEE Trans. Image Process. 20(6): 1709-1724

Parameswaran V, Singh M and Ramesh V 2010 Illumination compensation based change detection using order consistency. In: IEEE Conference on Computer Vision and Pattern Recognition (CVPR), 19821989

Sen-Ching S, Cheung and Chandrika Kamath 2004 Robust techniques for background subtraction in urban traffic video. In: IS\&T/SPIE Symposium on electronic imaging, San Jose, CA, US

Shahrizat Shaik Mohammed, Nooritawati Nd Tahir and Ramli Adnan 2010 background modeling and background subtraction performance for object detection. IEEE $6^{\text {th }}$ International Colloquium on Signal Processing and its Applications, 236-241

Shengyong Chen, Jianhua Zhang, Youfu Li and Jianwei Zhang 2012 A hierarchical model incorporating segmented regions and pixel descriptors for video background subtraction. IEEE Trans. Ind. Inform. 8: $118-127$

Shinya Miyamoori, Kanzunori Saito, Yohei Fukumizu, Hironori Yamauchi and Ritsumeikan 2011 Adaptive BP - RRC Mixture model for background subtraction. $4^{\text {th }}$ International Congress on Image and Signal Processing, 1180-1183

Songyin Fu, Gangyi Jiang and Mei Yu 2010 An effective background subtraction method based on pixel change classification. IEEE Int. Conf. Electrical and Control Eng. 4634-4637

Stauffer C and Grimson W 1999a Adaptive background mixture models for real-time tracking. IEEE Comput. Soc. 2: 2246-2252

Stauffer C and Grimson W E L 1999b Adaptive mixture models for real time tracking. In: Proceedings of IEEE, CVPR, 246-252

Syed Shazali S T, Cheong W L, Mohammaddan S, Abg Kamaruddin M N and Yassin A 2011 Motion detection using periodic background estimation subtraction method. $7^{\text {th }}$ International Conference on IT in Asia

Vijverberg J A, Loomans M J and Koeleman C J 2009 Global illumination compensation for back-ground subtraction using Gaussian-based background difference modeling. IEEE Conf. Adv. Video Signal Based Surveill. 0: 448-453

Weihua Xiong, Jumbin Guan and Haipeng Pan 2010 A new algorithm for moving objects detection based on background and consecutive frames subtraction, Proceedings of the $8^{\text {th }}$ World Congress on Intelligent Control and Automation July 6-9, Jinan, China

Yongquan Xia, Shaohui Ning, Han Shen, Zengzhou and Zhen Zhou 2010 Moving target detection algorithm based on background subtraction and frames subtraction. $2^{\text {nd }}$ Int. Conf. Industrial Mechatronics and Automation, 122-125 